\title{
Effects of carbonated liquid on swallowing dysfunction in dementia with Lewy bodies and Parkinson's disease dementia
}

This article was published in the following Dove Press journal:

Clinical Interventions in Aging

8 August 2017

Number of times this article has been viewed

\author{
Victoria Larsson' \\ Gustav Torisson ${ }^{1,2}$ \\ Margareta Bülow ${ }^{3}$ \\ Elisabet Londos' \\ 'Clinical Memory Research Unit, \\ Department of Clinical Sciences \\ Malmö, Lund University, ${ }^{2}$ Department \\ of Infectious Diseases, ${ }^{3}$ Diagnostic \\ Centre of Imaging and Functional \\ Medicine, Skåne University Hospital, \\ Malmö, Sweden
}

Background: Swallowing dysfunction is an increasingly recognized problem in patients with dementia with Lewy bodies (DLB) and Parkinson's disease dementia (PDD), which can result in aspiration pneumonia and death. Few studies have examined potential ways of improving swallowing function in this fragile patient group. The aim of this study was to evaluate swallowing dysfunction and carbonated liquid using videofluoroscopy in DLB and PDD patients. Methods: A total of 48 patients with DLB and PDD were referred for a clinical examination with videofluoroscopy. Descriptive overall assessments were provided at the time of the examination regarding swallowing function and the effects of different modifications, including carbonated thin liquid (CTL). Additionally, a repeated measures quantitative retrospective analysis has been performed comparing 1) thin liquids; 2) thickened liquids and 3) CTLs, with regard to the quantitative variables 1) pharyngeal transit time (PTT); 2) pharyngeal retention and 3) tracheal penetration.

Results: In all, 40/48 (83\%) of the patients had a swallowing dysfunction, which was confirmed on videofluoroscopy, with 34/40 (85\%) patients having a pharyngeal-type dysfunction. A total of $14 / 40(35 \%)$ patients with an objective swallowing impairment did not have any subjective swallowing symptoms. Out of the patients with swallowing dysfunction, $87 \%$ had an overall improved swallowing function with carbonated liquid. PTT for carbonated liquid (median $633 \mathrm{~ms}$, interquartile range [IQR] 516-786 ms) was quicker than for thin liquid (760 ms, IQR 613-940 ms, $P=0.014$ ) and thickened liquid ( $880.0 \mathrm{~ms}$, IQR $600-1,500 \mathrm{~ms}, P<0.001)$. No significant effect was seen in residue or penetration.

Conclusion: The majority of patients with DLB or PDD had a swallowing dysfunction, sometimes without subjective swallowing symptoms, which improved with carbonated liquid. This highlights the importance of investigating patients with videofluoroscopy and to carry out a prospective interventional study to further evaluate carbonated liquid, also addressing the effects on quality of life, aspiration and mortality.

Keywords: therapeutics, video recording, deglutition, dysphagia, carbonated beverages, swallowing disorders

\section{Introduction}

Globally, 47 million people are affected by dementia, ${ }^{1}$ and out of them, $15 \%-20 \%$ are estimated to have Lewy body dementia, 2,3 a term encompassing both dementia with Lewy bodies (DLB) and Parkinson's disease dementia (PDD). These neurodegenerative disorders share the same pathological hallmarks, with alpha-synuclein inclusions and neuronal loss, leading to complex clinical manifestations with cognitive dysfunction, motor disturbances, fluctuations, visual hallucinations and autonomic dysfunction. ${ }^{4}$
Correspondence: Victoria Larsson Clinical Memory Research Unit, Department of Clinical Sciences Malmö, Skåne University Hospital, Simrisbanvägen 14, 20502 Malmö, Sweden

Tel +46 40335517

Email victoria.larsson@med.lu.se 
There are no prevention or disease-modifying drugs, and consequently, treatment is directed toward symptom improvement. With quality of life being worse than other dementias, ${ }^{5}$ other pharmacological or non-pharmacological approaches are highly desired in this fragile patient group.

Swallowing is a complex sensorimotor task that is often impaired in neurodegenerative disorders, leading to serious health consequences and decreased quality of life. ${ }^{6}$ Patients with DLB have been found to have a high prevalence of subjective dysphagia, ${ }^{7}$ and the onset of dysphagia has been related to a shorter survival compared to other synucleinopathies. ${ }^{8}$ Pathological correlates have been identified with widespread alpha-synuclein deposits in brainstem areas responsible for swallowing function. ${ }^{9,10}$ Videofluoroscopy is the examination of choice to characterize dysphagia and has identified that patients with DLB often have a pharyngeal-type dysfunction, which increases risk of aspiration into the airways. ${ }^{11} \mathrm{~A}$ full evaluation of swallowing function and subsequent recommendations are therefore important in this patient group.

The current management for patients with swallowing dysfunction has focused on diet modification, head positioning and swallowing maneuvers, something which can be difficult for patients with cognitive impairment. Novel therapies are exploring stimulation of chemesthetic receptors, which can prevent aspiration by the activation of preventative reflexes. ${ }^{12}$ A way of achieving chemesthetic stimulation is to carbonate thin liquids, activating receptors by a carbonic anhydrase mechanism. ${ }^{13}$ A study of healthy volunteers using transcranial stimulation has shown that carbonated liquid increases the corticobulbar excitability of the pharyngeal projection with effects lasting up to 60 minutes, suggesting a modulatory pathway. ${ }^{14} \mathrm{~A}$ few studies have been done in clinical patients to assess the biomechanics or safety of carbonated liquid, ${ }^{15-18}$ but none as of yet have evaluated the role of carbonated liquids in DLB or PDD.

The aims of this study were to describe swallowing dysfunction in DLB and PDD patients and investigate if carbonated liquid can be helpful for these patients. We did a retrospective analysis, using descriptive and quantitative measures, evaluating patients with DLB and PDD who had carried out a therapeutic videoradiographic swallowing study (TVSS), where carbonated liquid was tested as part of the routine examination. Our hypothesis was that carbonated liquid would improve swallowing when compared to thin and thickened liquid, which would be relevant for clinicians and health care personnel caring for Lewy body dementia patients.

\section{Methods}

\section{Participants}

We used the electronic patient system to identify all patients who had been referred to the Diagnostic Centre of Imaging and Functional Medicine at the Skane University Hospital from the Memory Clinic in Malmö for a TVSS as part of clinical practice from 2006 to 2016. Only patients who had a diagnosis of DLB as defined by the consensus criteria from $2005^{3}$ or the diagnosis of $\mathrm{PDD}^{19}$ were included in the study. The diagnosis was confirmed by reviewing the electronic medical records. Patients with other diagnoses were excluded. There were no exclusions regarding cognitive level, comorbidities or treatment. If patients had multiple examinations, only the first one was used.

All patients were included in clinical follow-up programs at the Memory Clinic. Information about patient age, sex, duration of diagnosis, living situation, medications and previous history of pneumonia was collected from the electronic medical records to characterize the patient population.

To be able to assess the proportion of patients referred for a swallowing study, the number of patients with a diagnosis of DLB or PDD at the Memory Clinic in Malmö between 2006 and 2016 was estimated using the electronic patient system (using ICD-10 codes F02.8 G31.8A and F02.3 G20.9).

In this retrospective study, only regular clinical data were reviewed. All patients were included in longitudinal follow-up programs and had previously consented to prospective studies using the very same clinical data. At the time of conducting the study, $70 \%$ were deceased and thus not able to give further consent. In view of this, the local institutional review board (IRB) did not consider further consent to be applicable for this retrospective study but instead recommended an opt-out strategy consisting of an advertisement in a local newspaper. The Regional Ethical Review Board in Lund, Sweden, approved this study.

\section{Overall study design}

All patients carried out a TVSS according to a set of protocols as part of clinical practice (mentioned later). After the examination, the responsible speech and language pathologist (SLP) provided a descriptive assessment concerning the examination in full, ie, all swallows and all consistencies tested for that patient. Comments were made about what type of swallowing dysfunction is present and what modifications, including carbonated liquid, improved the swallowing function. These statements were returned to the referring clinician. 
In the first part of the study, we reviewed the descriptive statements with regard to overall swallowing dysfunction and global effect of carbonated liquid on the swallowing, referred to as the descriptive analysis. The second part of the study was a retrospective analysis of the videographs using quantitative measures (described later), referred to as the quantitative analysis.

\section{TVSS procedure}

Videofluoroscopy is a radiological investigation, recording moving images, while the patient is swallowing a radiopaque bolus, allowing visualization of bolus passage through the oral cavity, pharynx and esophagus. The images are transferred to a recording system, allowing repeated viewings of the images. A TVSS, sometimes called a modified barium swallow, is performed to be able to analyze the effects of different modified textures, solids and liquids and therapeutic strategies on physiology of swallowing.

The Diagnostic Centre of Imaging and Functional Medicine at Skane University Hospital has a set of protocols for TVSS that is used for both clinical practice and research purposes. The procedure is carried out by a radiologist and an SLP. Patients are examined in the seated upright position, in their wheelchair if necessary, and the examination always starts with a lateral projection to allow visualization of reference points and the bolus passage through swallowing structures. If possible, every patient is also examined with a frontal view to analyze any asymmetry and to follow at least one swallow through the esophagus to document free passage. However, the TVSS examination is focused on oral and pharyngeal swallowing function.

Patients are presented with different materials by a departmental health care assistant or rarely nursing home personnel accompanying the patient if needed for compliance and are told to swallow freely and completely whenever ready. The order of the materials is generally smooth fruit pudding, smooth puree (fish or meat), thick pâté, chopped normal food, thickened liquids, CTLs and thin liquids, but this sometimes varies due to the condition of the patient. The liquids are given in doses of 3 and $5 \mathrm{~mL}$, and the patients are also encouraged to drink freely if possible.

All test materials are prepared and mixed with barium sulfate in the hospital kitchen and stored in a freezer in the radiological department until used, except carbonated liquid, which is mixed at the time of use. Solid bolus consisted of $45 \mathrm{~g}$ product and $15 \mathrm{~g}$ barium sulfate (E-Z-HD barium sulfate suspension $98 \%$ w/w, produced by E-Z-EM Canada
Inc., Lake Success, NY, USA, and distributed by Tamro, Stockholm, Sweden). One portion of thickened liquid consisted of $100 \mathrm{~g}$ of fruit puree mixed with $30 \mathrm{mg}$ E-Z-HD suspension, thin liquid consisting of water with E-Z-HD suspension $40 \%$ weight/volume and CTL consisting of water with E-Z-HD suspension $40 \%$ weight/volume mixed with $4 \mathrm{mg}$ of sodium bicarbonate $/ 100 \mathrm{~mL}$ barium contrast.

The examination was performed using digital radiological equipment. The average time for the radiological exposure was 3 minutes with a corresponding radiation dose of 2-5 mSv. The examination was transmitted digitally to an electronic picture archiving and communication system, producing 16 frames per second (f/s), equating to 1 frame/62.5 ms, allowing frame-by-frame slow-motion analysis after the examination. Examinations since 2011 and onward have been archived using the software PharyDoc ${ }^{\mathbb{B}}$ (Medical Imaging Technologies, Lund, Sweden) and could therefore be used for retrospective quantitative analysis.

\section{Quantitative videographic analysis}

Videofluoroscopy allows measurements of temporal parameters in the different swallowing phases and measurements of retention and penetration. To assess the effects of CTL, we wanted to compare it with thin liquid and thickened liquid using quantitative measures. Therefore, a retrospective quantitative analysis was carried out by authors VL and MB using archived videoradiographic examinations.

For each patient, the first swallow of each consistency (thin liquid, thickened liquid and carbonated liquid) was analyzed, ie, three swallows in total per patient. The first swallow was used, as this is often clinically recognized as the poorest swallow in this patient group. Videos were analyzed at a normal speed and in slow motion, as many times as needed for a confident judgment by MB who is experienced in TVSS. There was no blinding of consistencies, with carbonated liquid being identified by the air bubbles on TVSS.

The variables measured during the quantitative analysis were pharyngeal transit time (PTT), pharyngeal retention and penetration. PTT was defined as the time (in milliseconds) from when the apex of the bolus crossed the level of the faucial isthmus to when the peristaltic wave left the cricopharyngeal muscle. Pharyngeal residue was defined as retention of material in the valleculae and/or pyriform sinuses. This was scored using a protocol grading residue severity in relation to the perceived height of the contrast material considering the surrounding space (Table 1) ${ }^{20}$ Penetration was defined as the entrance of bolus material into the airway and graded 
Table I Grading for pharyngeal residue scale and penetration scale

\begin{tabular}{l}
\hline Pharyngeal residue scale \\
I No residue \\
2 Mild residue \\
3 Moderate \\
4 Severe \\
Penetration scale \\
I No penetration \\
2 Subepiglottic penetration (just below the epiglottis) \\
3 Supraglottic penetration (above the true vocal cords) \\
4 Tracheal penetration (below the true vocal cords) \\
\hline
\end{tabular}

using a departmental protocol similar to the PenetrationAspiration Scale. ${ }^{21}$

\section{Statistical methods}

The data analysis of the quantitative measures was done using SPSS (Version 22; IBM Corporation, Armonk, NY, USA). We used a repeated measures design with three liquid consistencies measured for each patient. The primary statistical analysis was to measure the comparison of change for the different liquid consistencies within subjects using the Wilcoxon signed-rank test. This was preceded by the Friedman test, a non-parametric alternative to the analysis of variance (ANOVA) of repeated measures. Comparison of change in PTT between groups of patients (DLB and PDD, men and women) was done using the Mann-Whitney $U$ test. Spearman's rank order correlation was used for testing nonparametric association.

Effect sizes were calculated using the formula $r=Z / \sqrt{ } N{ }^{22}$ All $P$-values were two tailed, and statistical significance was set at $P \leq 0.05$. The primary analysis was ANOVA for three independent groups, and with a Bonferroni correction applied, the resulting significance level was $<0.017$.

\section{Results}

Between 2006 and 2016, 66 TVSS referrals were done from the Memory Clinic in Malmö. Out of these, 15 patients had diagnoses other than DLB or PDD, with one being a repeat, and they were excluded. Of the remaining 51 referrals, three were repeat examinations and were therefore excluded. Out of the 48 patients remaining for analysis, there were 38 DLB patients and 10 PDD patients. The total number of patients with a diagnosis of DLB or PDD at the Memory Clinic in Malmö between 2006 and 2016 was 382 patients (274 DLB patients and 108 PDD patients).

The baseline demographics have been described in Table 2. One patient had a history of a stroke, but this was
Table 2 Baseline characteristics

\begin{tabular}{llll}
\hline & All patients & DLB $(\mathbf{n}=\mathbf{3 8})$ & PDD $(\mathbf{n}=\mathbf{l 0})$ \\
\hline Age (years) & $76.0( \pm 6.8)$ & $75.6( \pm 6.8)$ & $77.5( \pm 6.8)$ \\
Male & 62.5 & 60.5 & 70 \\
Duration of diagnosis & $21(4-40)$ & $17.5(4-39)$ & $35(6-52)$ \\
at TVSS (months) & & & \\
MMSE & $19.3( \pm 5.9)$ & $19.5( \pm 6.1)$ & $18.6( \pm 5.4)$ \\
Living at home & 85.4 & 86.8 & 80 \\
AChEi treatment & 87.5 & 89.5 & 80 \\
Levodopa treatment & 50 & 39.5 & 90 \\
Levodopa dose (mg) & $300(200-575)$ & $300(100-300)$ & $600(400-838)$ \\
Memantine treatment & 66.7 & 65.8 & 70 \\
History of pneumonia & 43.8 & 44.7 & 40 \\
\hline
\end{tabular}

Note: Values are in mean $( \pm S D)$, median $(I Q R)$ and percentages.

Abbreviations: DLB, dementia with Lewy bodies; PDD, Parkinson's disease dementia; TVSS, therapeutic videoradiographic swallowing study; MMSE, Mini-Mental State Examination; $\mathrm{AChEi}$, acetylcholinesterase inhibitors; IQR, interquartile range.

not the referral reason. No patients had a history of head or neck tumors, surgery to the head or neck regions or peripheral nerve disease.

\section{Descriptive swallowing assessments}

The assessments have been summarized in Table 3, including referral reasons, type of swallowing dysfunction and response to carbonated liquid. A confirmed swallowing dysfunction was found in 40 out of 48 patients, with a pharyngeal-type dysfunction seen in 34 patients. Out of the 40 patients with swallowing dysfunction, 14 patients did not complain of swallowing problems. Across the entire swallowing examination,

Table 3 Summary of descriptive statements from TVSS

\begin{tabular}{|c|c|c|c|}
\hline & $\begin{array}{l}\text { All } \\
(n=48)\end{array}$ & $\begin{array}{l}\text { DLB } \\
(n=38)\end{array}$ & $\begin{array}{l}\text { PDD } \\
(n=10)\end{array}$ \\
\hline \multicolumn{4}{|l|}{ Reason for referral, n (\%) } \\
\hline Subjective swallowing difficulties & $32(67)$ & $23(6 I)$ & $9(90)$ \\
\hline Cough only & $9(19)$ & 24 & $0(0)$ \\
\hline Unable to straighten neck & $\mathrm{I}(2)$ & I (3) & $0(0)$ \\
\hline Excess saliva & $\mathrm{I}(2)$ & I (3) & $0(0)$ \\
\hline Clearing throat & I (2) & I (3) & $0(0)$ \\
\hline History of pneumonia & $2(4)$ & I (3) & I (I0) \\
\hline No symptoms but other clinical suspicion & $2(4)$ & $2(5)$ & $0(0)$ \\
\hline $\begin{array}{l}\text { Swallowing dysfunction confirmed } \\
\text { on TVSS, } \mathbf{n}(\%)\end{array}$ & $40(83)$ & $31(82)$ & $9(90)$ \\
\hline \multicolumn{4}{|c|}{ Type of swallowing dysfunction observed, n (\%) } \\
\hline Oral dysfunction only & $4(8)$ & $4(\mathrm{II})$ & $0(0)$ \\
\hline Pharyngeal dysfunction only & $24(50)$ & $19(50)$ & $5(50)$ \\
\hline Combined oropharyngeal dysfunction & $10(21)$ & $7(18)$ & $3(30)$ \\
\hline Pharyngeal retention & $24(50)$ & $19(50)$ & $5(50)$ \\
\hline Tracheal penetration & $13(27)$ & $9(24)$ & $4(40)$ \\
\hline $\begin{array}{l}\text { Improved swallowing with } \\
\text { carbonated liquid,* n (\%) }\end{array}$ & $34(87)$ & $27(87)$ & $7(88)$ \\
\hline
\end{tabular}

Note: *One patient with confirmed swallowing dysfunction not tried on carbonated liquid $(n=39)$.

Abbreviations: TVSS, therapeutic videoradiographic swallowing study; DLB, dementia with Lewy bodies; PDD, Parkinson's disease dementia. 
24 patients showed pharyngeal retention and 13 patients had evidence of tracheal penetration. Nine patients with a confirmed retention and two of those with tracheal penetration did not have subjective swallowing problems. Out of those with a swallowing dysfunction, carbonated liquid was found to improve the global swallowing in 34 out of 39 patients. DLB and PDD patients improved similarly with carbonated liquid.

\section{Quantitative analysis}

Videoradiographic recordings prior to 2011 were not archived and therefore not available for analysis, and a further two examinations were missing in the system. Thus, out of the 48 patients, 25 patients were included in the quantitative analysis.

Assessing PTT using the Friedman test, there was a statistically significant difference depending on which type of liquid was used, $\chi^{2}(2 \mathrm{df})=12.645, P=0.002$. Post hoc analysis with Wilcoxon signed-rank tests was conducted with a Bonferroni correction applied, resulting in a significance level set at $P<0.017$. Median (interquartile range [IQR]) PTT (ms) values for thin, thick and carbonated liquids were 760 (613-940), 880 (600-1,500) and 633 (516-786), respectively. There were no significant differences between the thick liquid and thin liquid $(Z=-1.841, P=0.066, r=-0.27$ ). However, there was a significant difference between carbonated liquid and thin liquid $(Z=-2.464, P=0.014, r=-0.36)$ and carbonated liquid and thick liquid $(Z=-3.499, P<0.001$, $r=-0.51)$. Comparing diagnoses or sex did not reveal any significant differences. There was no association between PTT and levodopa dose.

Retention and penetration was measured with the scales described in the Methods section. Out of the 25 patients, 11 subjects had an abnormality in at least one of the three swallows studied on either residue or penetration (Table S1). Thickened liquid worsened the degree of residue in four patients compared to thin liquid. Carbonated liquid improved the severity of pharyngeal retention in six out of nine patients compared to thin or thickened liquid. One patient had worse retention with carbonated liquid compared to thin liquid. The depth of penetration was improved with carbonated liquid in the three patients with observed penetration on thin or thick liquid.

\section{Discussion}

This study is the first to evaluate the role of carbonated liquid for swallowing dysfunction in patients with DLB or PDD. Our main findings show that swallowing dysfunction is common and that carbonated liquid could improve swallowing function and therefore should be evaluated as a useful and simple non-pharmacological therapy in this patient group.

From the descriptive analysis, swallowing dysfunction was confirmed in $83 \%$ of the patients referred for TVSS. A pharyngeal-type dysfunction or combined oropharyngeal dysfunction was seen in $71 \%$ of the patients, while only $8 \%$ had a purely oral dysfunction. Notably, some patients with a confirmed pharyngeal retention or aspiration did not have any subjective swallowing complaints, which is in keeping with previous findings in patients with DLB and PD. ${ }^{23-25}$

In those patients with a swallowing dysfunction on videofluoroscopy, $87 \%$ were found to have an improved overall swallowing function with carbonated liquids (Table 3 ). In the quantitative analysis, PTT was significantly improved with carbonated liquid compared to both thin and thickened liquids with a medium and large effect size, respectively $(P=0.014$, $r=-0.36$ and $P<0.0001, r=-0.51$, respectively). Although an improved PTT alone cannot indicate a safe swallow, it is a relevant measure as it has previously been shown that PTT is prolonged in PD patients with a history of aspiration pneumonia. ${ }^{26}$

The descriptive assessment showed that $27 \%$ of patients had evidence of tracheal penetration at some stage of the examination. This is similar to rates seen in another videofluoroscopic study of $\mathrm{PD}$ patients. ${ }^{23} \mathrm{We}$ also found that $50 \%$ of patients had pharyngeal retention, something which increases aspiration risk. ${ }^{20}$ To further assess this, we also intended to compare the severity of retention and depth of penetration between thin, thickened and carbonated liquids using quantitative measures. However, by only looking at one swallow for each of the consistencies, it was apparent that we did not fully capture penetration and retention to the same extent as the overall descriptive assessment of the full examination. Nevertheless, carbonated liquid did improve the severity of retention and depth of penetration in certain individuals (Table S1), suggesting that it could be useful for contributing to a safer swallow. This is also in line with our clinical observations, where we frequently see that patients with a pharyngeal residue often can clear this when administered carbonated liquid (Video S1). Notably, thickened liquid, which is sometimes used as a modification in swallowing dysfunction, appeared to increase pharyngeal retention. This is similar to previous observations that higher viscosity liquids has a heightened risk of post-swallow pharyngeal residue, although probably still reducing penetration overall. ${ }^{27}$

This is the first study that specifically assessed liquid modification for DLB and PDD patients. The major advantage 
is that our study population was relevant for a memory clinic population, consisting of a heterogeneous group by not excluding patients with certain comorbidities or clinical features. We used a set of routine TVSS protocols, and there were only two experienced SLPs involved in providing the descriptive assessments, reducing the variation between the assessments. The quantitative analysis has been conducted according to standardized variables used in other swallowing studies, allowing for comparison.

There are a number of limitations of this study. First, the retrospective nature meant that we were unable to influence the conducted examination, such as the technical aspects or randomizing the stimulus order. The frame rate used was limited to $16 \mathrm{f} / \mathrm{s}$, which might have resulted in instances of missed penetration and contributed to coarse measurement of the PTT. Moreover, we were limited by lacking another experienced TVSS researcher in order to carry out reliability testing to validate the quantitative measures.

Despite these weaknesses, our findings are clinically relevant, and we are therefore encouraging future studies that could address the abovementioned issues, including a higher frame rate of $30 \mathrm{f} / \mathrm{s}$ to more clearly identify swallowing impairment, ${ }^{28}$ and potentially more extensive quantitative measures. ${ }^{29}$ When comparing carbonated liquid to other consistencies, it should possibly be the last liquid to be administered in the study protocol, since carbonated liquid has been shown to have a positive effect on corticobulbar excitability, ${ }^{14}$ which could potentially influence the performance of subsequent swallows. ${ }^{14}$ Importantly, our current assessments are concerning immediate effects that do not necessarily indicate any long-term effectiveness, and therefore, extended follow-up is needed to evaluate the impact also on overall health, including quality of life, pneumonia incidence and mortality.

Silent aspiration is a risk factor for pneumonia and early death, ${ }^{30}$ and our research finding further highlights the importance of clinicians and other health care professionals to suspect swallowing dysfunction, even in cases where there are no subjective swallowing problems. For example, unintentional weight loss or recurrent pneumonias should prompt an appropriate swallowing examination, preferably with videofluoroscopy. In our own Memory Clinic, only $12.5 \%$ of patients with DLB or PDD were referred for a swallowing study, which could possibly be because of an underrecognition of swallowing problems, something which could perhaps be improved by carrying out a routine swallowing screen for these patients.

A degree of patient cooperation is needed for TVSS, which could be influenced by severe cognitive impairment.
This is not something experienced in the current study, which might be because all patients were referred by their responsible clinician and an individual assessment regarding the ability to cooperate would have already taken place prior to coming to the examination.

Although carbonated liquid is generally safe, this is not the case for all patients. In our clinical experience, some patients can have a negative response, resulting in tracheal penetration, particularly if the patient has an incomplete pharyngeal swallow. This was seen in one patient in another study, ${ }^{18}$ which is why each patient still needs an individual assessment to ensure a positive and safe swallow response to carbonated liquid prior to clinical recommendation.

We did not use a control group in this study, as the primary aim was not to show that carbonated liquid is better in DLB or PDD patients than in other groups. On contrary, we believe that the results could be of relevance to other patient groups, including patients with Parkinson's disease without cognitive symptoms, where dysphagia is an early and sometimes asymptomatic feature, ${ }^{31,32}$ and carbonated liquid might be similarly or even more beneficial. This should be considered particularly as carbonated liquid is generally well tolerated ${ }^{17}$ compared to less palatable diet modification, which is important in chronic disease as it might allow for better compliance and higher patient satisfaction. It might also reduce the risk of dehydration, something that is sometimes seen with thickened liquids. ${ }^{27}$

\section{Conclusion}

We believe that by assessing DLB and PDD patients with TVSS, it is possible to visualize swallowing dysfunctions that are common and sometimes asymptomatic, providing important information for patient management. It also allows evaluation of various treatment strategies and modifications, where we believe that carbonated liquid should be considered as an alternative, although we need prospective interventional studies to confirm the effect. Nevertheless, if carbonated liquid is found to be beneficial after individual assessment, we believe that it could be recommended as a complement in clinical practice, which is important as therapeutic options are limited. Further studies are needed to address the long-term outcomes on quality of life, aspiration risk and mortality.

\section{Acknowledgments}

Many thanks to Professor Lennart Minthon for facilitating the research environment at the Memory Clinic and professor Olle Ekberg for his research support and radiological skills at the Diagnostic Centre of Imaging and Functional Medicine. This study was supported by the Swedish Research 
Council (grant no 523-2010-520) and by Lions Research Foundation Skane.

\section{Disclosure}

The authors report no conflicts of interest in this work.

\section{References}

1. Prince M, Wimo A, Guerchet M, Ali GC, Wu Y, Prina AM. World Alzheimer Report 2015: The Global Impact of Dementia. An Analysis of Prevalence, Incidence, Costs and Trends. London: Alzheimer's Disease International; 2015. Available from: https://www.alz.co.uk/ research/world-report-2015. Accessed March 3, 2016.

2. Zaccai J, McCracken C, Brayne C. A systematic review of prevalence and incidence studies of dementia with Lewy bodies. Age Ageing. 2005; 34(6):561-566.

3. McKeith IG, Dickson DW, Lowe J, et al. Diagnosis and management of dementia with Lewy bodies: third report of the DLB Consortium. Neurology. 2005;65(12):1863-1872.

4. Lippa CF, Duda JE, Grossman M, et al. DLB and PDD boundary issues: diagnosis, treatment, molecular pathology, and biomarkers. Neurology. 2007;68(11):812-819.

5. Bostrom F, Jonsson L, Minthon L, Londos E. Patients with dementia with Lewy bodies have more impaired quality of life than patients with Alzheimer disease. Alzheimer Dis Assoc Disord. 2007;21(2): $150-154$

6. Wirth R, Dziewas R, Beck AM, et al. Oropharyngeal dysphagia in older persons - from pathophysiology to adequate intervention: a review and summary of an international expert meeting. Clin Interv Aging. 2016;11:189-208.

7. Shinagawa S, Adachi H, Toyota Y, et al. Characteristics of eating and swallowing problems in patients who have dementia with Lewy bodies. Int Psychogeriatr. 2009;21(3):520-525.

8. Muller J, Wenning GK, Verny M, et al. Progression of dysarthria and dysphagia in postmortem-confirmed parkinsonian disorders. Arch Neurol. 2001;58(2):259-264.

9. Seidel K, Mahlke J, Siswanto S, et al. The brainstem pathologies of Parkinson's disease and dementia with Lewy bodies. Brain Pathol. 2015;25(2):121-135.

10. Kovari E, Horvath J, Bouras C. Neuropathology of Lewy body disorders. Brain Res Bull. 2009;80(4-5):203-210.

11. Londos E, Hanxsson O, Alm Hirsch I, Janneskog A, Bulow M, Palmqvist S. Dysphagia in Lewy body dementia - a clinical observational study of swallowing function by videofluoroscopic examination. BMC Neurol. 2013;13:140.

12. Logemann JA, Pauloski BR, Colangelo L, Lazarus C, Fujiu M, Kahrilas PJ. Effects of a sour bolus on oropharyngeal swallowing measures in patients with neurogenic dysphagia. J Speech Hear Res. 1995; 38(3):556-563.

13. Dessirier JM, Simons CT, Carstens MI, O’Mahony M, Carstens E. Psychophysical and neurobiological evidence that the oral sensation elicited by carbonated water is of chemogenic origin. Chem Senses. 2000;25(3): 277-284.

14. Elshukri O, Michou E, Mentz H, Hamdy S. Brain and behavioral effects of swallowing carbonated water on the human pharyngeal motor system. J Appl Physiol (1985). 2016;120(4):408-415.
15. Bulow M, Olsson R, Ekberg O. Videoradiographic analysis of how carbonated thin liquids and thickened liquids affect the physiology of swallowing in subjects with aspiration on thin liquids. Acta Radiol. 2003;44(4):366-372.

16. Morishita M, Mori S, Yamagami S, Mizutani M. Effect of carbonated beverages on pharyngeal swallowing in young individuals and elderly inpatients. Dysphagia. 2014;29(2):213-222.

17. Sdravou K, Walshe M, Dagdilelis L. Effects of carbonated liquids on oropharyngeal swallowing measures in people with neurogenic dysphagia. Dysphagia. 2012;27(2):240-250.

18. Lundine JP, Bates DG, Yin H. Analysis of carbonated thin liquids in pediatric neurogenic dysphagia. Pediatr Radiol. 2015;45(9):1323-1332.

19. Emre M, Aarsland D, Brown R, et al. Clinical diagnostic criteria for dementia associated with Parkinson's disease. Mov Disord. 2007;22(12): 1689-1707; quiz1837.

20. Eisenhuber E, Schima W, Schober E, et al. Videofluoroscopic assessment of patients with dysphagia: pharyngeal retention is a predictive factor for aspiration. AJR Am J Roentgenol. 2002;178(2):393-398.

21. Rosenbek JC, Robbins JA, Roecker EB, Coyle JL, Wood JL. A penetration-aspiration scale. Dysphagia. 1996;11(2):93-98.

22. Rosenthal R. Parametric measures of effect size. In: Cooper H, Hedges L, editors. The Handbook of Research Synthesis. New York: Russell Sage Foundation; 1994:231-244.

23. Yamamoto T, Kobayashi Y, Murata M. Risk of pneumonia onset and discontinuation of oral intake following videofluorography in patients with Lewy body disease. Parkinsonism Relat Disord. 2010; 16(8):503-506.

24. Bird MR, Woodward MC, Gibson EM, Phyland DJ, Fonda D. Asymptomatic swallowing disorders in elderly patients with Parkinson's disease: a description of findings on clinical examination and videofluoroscopy in sixteen patients. Age Ageing. 1994;23(3):251-254.

25. Monteiro L, Souza-Machado A, Pinho P, Sampaio M, Nobrega AC, Melo A. Swallowing impairment and pulmonary dysfunction in Parkinson's disease: the silent threats. J Neurol Sci. 2014;339(1-2): 149-152.

26. Lin CW, Chang YC, Chen WS, Chang K, Chang HY, Wang TG. Prolonged swallowing time in dysphagic Parkinsonism patients with aspiration pneumonia. Arch Phys Med Rehabil. 2012;93(11): 2080-2084.

27. Steele CM, Alsanei WA, Ayanikalath S, et al. The influence of food texture and liquid consistency modification on swallowing physiology and function: a systematic review. Dysphagia. 2015;30(1):2-26.

28. Bonilha HS, Blair J, Carnes B, et al. Preliminary investigation of the effect of pulse rate on judgments of swallowing impairment and treatment recommendations. Dysphagia. 2013;28(4):528-538.

29. Ellerston JK, Heller AC, Houtz DR, Kendall KA. Quantitative measures of swallowing deficits in patients with Parkinson's disease. Ann Otol Rhinol Laryngol. 2016;125(5):385-392.

30. Pikus L, Levine MS, Yang YX, et al. Videofluoroscopic studies of swallowing dysfunction and the relative risk of pneumonia. AJR Am J Roentgenol. 2003;180(6):1613-1616.

31. Nilsson H, Ekberg O, Olsson R, Hindfelt B. Quantitative assessment of oral and pharyngeal function in Parkinson's disease. Dysphagia. 1996; 11(2):144-150.

32. Potulska A, Friedman A, Krolicki L, Spychala A. Swallowing disorders in Parkinson's disease. Parkinsonism Relat Disord. 2003;9(6): 349-353. 


\section{Supplementary materials}

Table SI Comparison of grading on penetration and residue scales for different liquid consistencies in each patient with abnormal findings in quantitative analysis

\begin{tabular}{|c|c|c|c|c|c|c|c|c|c|c|c|c|}
\hline \multirow{2}{*}{$\begin{array}{l}\text { ID } \\
\text { no }\end{array}$} & \multicolumn{6}{|c|}{ Pharyngeal retention scale } & \multicolumn{6}{|c|}{ Penetration scale } \\
\hline & Thin & Thick & CTL & $\Delta$ thin-thick & $\Delta$ thin-CTL & $\Delta$ thick-CTL & Thin & Thick & CTL & $\Delta$ thin-thick & $\Delta$ thin-CTL & $\Delta$ thick-CTL \\
\hline 5 & 3 & 3 & 1 & 0 & -2 & -2 & 2 & 1 & 1 & -1 & -1 & 0 \\
\hline 7 & 1 & I & 1 & 0 & 0 & 0 & 2 & I & 1 & -1 & -1 & 0 \\
\hline 8 & I & 2 & 1 & +1 & 0 & -1 & 1 & I & 1 & 0 & 0 & 0 \\
\hline 15 & I & I & 1 & 0 & 0 & 0 & 1 & 2 & 1 & +1 & 0 & -1 \\
\hline 17 & 4 & 3 & 2 & -1 & -2 & -1 & 1 & I & 1 & 0 & 0 & 0 \\
\hline 18 & 2 & 2 & 1 & 0 & -1 & -1 & I & I & I & 0 & 0 & 0 \\
\hline 20 & I & 2 & - & +1 & - & - & I & I & - & 0 & - & - \\
\hline 21 & 1 & 2 & 1 & +1 & 0 & -1 & I & I & I & 0 & 0 & 0 \\
\hline 22 & 2 & 3 & 3 & +1 & +1 & 0 & 1 & I & 1 & 0 & 0 & 0 \\
\hline 23 & 2 & 2 & 2 & 0 & 0 & 0 & I & I & 1 & 0 & 0 & 0 \\
\hline 25 & 2 & 2 & 1 & 0 & -1 & -1 & I & 1 & I & 0 & 0 & 0 \\
\hline
\end{tabular}

Abbreviations: thin, thin liquid; thick, thick liquid; CTL, carbonated thin liquid.

Video SI Two sequences of a videographic swallowing examination showing an 82-year-old male swallowing (A) thin liquid, showing retention and aspiration, and (B) carbonated thin liquid improving the swallow.

Note: The sequences come from Margareta Bülow, Diagnostic Centre of Imaging and Functional Medicine, Skåne University Hospital, Malmö, Sweden.

\section{Publish your work in this journal}

Clinical Interventions in Aging is an international, peer-reviewed journal focusing on evidence-based reports on the value or lack thereof of treatments intended to prevent or delay the onset of maladaptive correlates of aging in human beings. This journal is indexed on PubMed Central, MedLine,
CAS, Scopus and the Elsevier Bibliographic databases. The manuscript management system is completely online and includes a very quick and fair peer-review system, which is all easy to use. Visit http://www.dovepress. com/testimonials.php to read real quotes from published authors. 